\title{
Targeting pericranial nerve branches to treat migraine: Current approaches and perspectives
}

$0(0) 1-15$

(C) International Headache Society 2015

Reprints and permissions:

sagepub.co.uk/journalsPermissions.nav DOI: 10.1 177/03331024155735। ।

cep.sagepub.com

@SAGE

\author{
Anna Ambrosini', Carmelo D'Alessio', Delphine Magis² and \\ Jean Schoenen ${ }^{2}$
}

\begin{abstract}
Background: Migraine is a highly prevalent neurological disorders and a major individual and societal burden. Migraine is not curable at the present time, but it is amenable to acute symptomatic and preventive pharmacotherapies.

Summary: Since the latter are frequently unsatisfactory, other treatment strategies have been used or are being explored. In particular, interventions targeting pericranial nerves are now part of the migraine armamentarium. We will critically review some of them, such as invasive and noninvasive neurostimulation, therapeutic blocks and surgical decompressions. Conclusions: Although current knowledge on migraine pathophysiology suggests a central nervous system dysfunction, there is some evidence that interventions targeting peripheral nerves are able to modulate neuronal circuits involved in pain control and that they could be useful in some selected patients. Larger, well-designed and comparative trials are needed to appraise the respective advantages, disadvantages and indications of most interventions discussed here.
\end{abstract}

\section{Keywords}

Migraine, treatment, surgery, neurostimulation, blocks, pericranial nerves

Date received: 21 July 20I4; revised: 8 September 20I4; accepted: 10 January 2015

\section{Introduction}

Migraine is one of the most common neurological disorders, affecting $18.5 \%$ of the general population (1), and it causes marked disability in many patients $(2,3)$. In its episodic form, it is characterized by recurrent attacks of moderate/severe headache associated with nausea and/or vomiting, photophobia and phonophobia (4). In $20-30 \%$ of patients the headache is preceded or accompanied at its onset by a sequence of reversible focal neurological symptoms called "aura." Classically it consists of a scintillating scotoma that can be followed by sensory and language disturbances and, in hemiplegic migraine, by motor symptoms.

Episodic migraine may evolve into chronic migraine when headache days exceed 15 per month with at least eight migraine headache days (4). Chronic migraine is the most disabling form of migraine and affects at least $0.5 \%$ of the general population (1).

\section{Pathophysiology}

Migraine is thought to be a central neurovascular disorder. The migraine headache is likely generated in the trigeminovascular system (TVS) (5) that can be activated by cortical spreading depression (CSD), i.e. slowly propagating waves of brief neuronal and glial depolarization followed by prolonged neuronal inactivation $(6,7)$, which is responsible for the migrainous aura. In migraine without aura the mechanisms underlying TVS activation are still controversial, but a role for dysfunctioning central pain control systems has been suggested (8). The migraine attack is a sequential process, comprising in $>30 \%$ of patients premonitory symptoms $(9,10)$ accompanied on neuroimaging by activation in the ventral hypothalamus (11).

The predisposition to attack recurrence in migraine is reflected in functional brain changes that fluctuate

'IRCCS NEUROMED, Pozzilli (Isernia), Italy

${ }^{2}$ Headache Research Unit, University of Liège, Citadelle Hospital, Belgium D.M. and J.S. contributed equally to this article.

Corresponding author:

Anna Ambrosini, Dept. Headache Medicine - IRCCS NEUROMED, Via Atinense, I8 - I 86077 - Pozzilli (Isernia), Italy.

Email: anna.ambrosini@neuromed.it 
over the migraine cycle, such as those related to the processing of sensory stimuli and cortical reactivity (12), and to mitochondrial energy metabolism (13-15). Migraine is supposed to be a complex polygenic disorder where the genetic load sets a threshold that is influenced by environmental and hormonal factors (16). In chronic migraine there are prominent changes in cerebral function and structure, many of which differ from those found in the episodic type (17). It has been suggested that in some chronic patients central sensitization amplifies and becomes permanent (18), which is probably the reason why many preventive therapies lose efficacy.

Migraine is thus chiefly a central nervous system disorder. There is nonetheless some circumstantial evidence that the peripheral nervous system might play a causal or aggravating role in certain migraine patients. For instance, pericranial tenderness on palpation is well known in migraineurs (19). In $30 \%$ of patients palpation of scalp trigger points may provoke an attack (20). In one patient a migrainous headache was suggested to be due to compression of the lesser occipital nerve by a lymph node (21). It was recently shown that trigeminovascular meningeal afferents project extracranially through the skull (22) and that activation of these extracranial afferents in rats causes release of calcitonin gene-related peptide (CGRP) from the dura, providing evidence that extracranial noxious signals may influence meningeal nociception (23). Despite the scarcity of data favoring a role for extracranial peripheral nerves in migraine, these nerves have been targeted by various therapeutic interventions.

\section{Management}

The management of migraine patients includes acute interventions to alleviate the attack and preventive treatments aiming at a reduction of attack frequency and disability. Acute antimigraine drugs comprise nonsteroidal anti-inflammatory drugs (NSAIDs), simple or combined analgesics and specific antimigraine drugs like triptans and, in some countries, ergots. Analgesics and specific antimigraine drugs have a high propensity to chronify migraine when overused (24). Preventive drugs have limited efficacy (on average $50 \%$ ). Many of them have cumbersome adverse effects (25) and they are likely to become progressively ineffective in chronic migraine (26) or medication-overuse headache (27).

Alternative treatments have been used for a long time, but they have received increasing attention recently, given the lack of new advances in pharmacotherapy.

We will focus here on interventions that are supposed to act on pericranial nerve branches.
Schematically they can be subdivided into neurostimulations, infiltrations/blocks, and surgical decompressions.

\section{Peripheral neurostimulation}

Electrical stimulation of peripheral nerves (PNS) is an effective way to relieve pain within the territory of the stimulated nerve. It is possibly due to the activation of afferent $A \beta$ fibers and subsequent gate control mechanisms in the spinal cord dorsal horn or/and to the activation of descending supraspinal controls from periaqueductal gray and rostroventromedial medulla $(28,29)$. PNS was initially used in chronic pain syndromes (30), thereafter in occipital neuralgia (31) and subsequently in migraine prevention (32). The modality of PNS was often chosen according to the migraine form, invasive - continuous and applied in the most severely disabled patients - or noninvasive-intermittent and applicable to all patients.

\section{Invasive PNS}

Invasive PNS was explored as preventive therapy quasiexclusively in patients suffering from drug-resistant chronic migraine.

Occipital nerve stimulation (ONS). The most studied technique is greater ONS. Besides small and/or heterogeneous open studies, three short-term (i.e. three months each) randomized, controlled trials (RCTs) were published (33-35). The Occipital Nerve Stimulation for the Treatment of Chronic Migraine Headache (ONSTIM) study ( $N=66$ patients) (34) showed a reduction of at least $50 \%$ in headache frequency and a decrease on the headache intensity scale in $39 \%$ of patients treated with active ONS during 12 weeks, whereas no improvement was found in the sham-stimulated or medically treated groups. However, ONSTIM was not powered to convincingly demonstrate effectiveness of ONS. In the sham-controlled Precision Implantable Stimulator for Migraine (PRISM) study (33), ONS did not produce any significant reduction in headache days in 125 patients with drug-resistant migraine with or without medication overuse. Finally, in the largest RCT of ONS in 157 patients with chronic migraine, no difference was found between sham and verum groups in the primary outcome measure (at least $50 \%$ reduction in mean daily headache intensity). However, a higher percentage of effectively stimulated patients achieved a $30 \%$ reduction in mean headache days $(p<0.05)$ and a decrease in migraine-related disability score (MIDAS) $(p<0.01) ; 51 \%$ of patients stated they 
were satisfied with the treatment compared to $19 \%$ in the sham group (35). After the three-month randomized phase, patients continued an open-label phase of 40 weeks. Monthly headache days were significantly reduced by 6.7 days in the intention-to-treat group, and by 7.7 days in a group of patients with "intractable" chronic migraine $(p<0.01)$ (36). In a recent small, randomized, crossover study of eight patients suffering from chronic migraine, suprathreshold stimulation was more effective than subthreshold stimulation, while the latter was superior to no stimulation (37).

These data suggest that ONS may be a promising treatment option for chronic migraine patients, but conclusive evidence from large RCTs is still missing. Moreover ONS can be associated with adverse effects and complications (local pain, intolerable paresthesias, local infection, electrode displacement, battery replacement) and the cost of the device is high (around 15,000-20,000 euros) while the clinical benefit seems modest (32).

In a retrospective open study of 44 patients with chronic migraine, the combination of ONS with supraorbital nerve stimulation (SNS) was reported to reduce the frequency of severe headaches by $81 \%(38,39)$. Half the patients had nearly complete disappearance of headaches (mean follow-up 13 months) (40), which is an interesting result that needs to be confirmed in an RCT.

Vagus nerve stimulation (VNS). Invasive VNS has shown efficacy in refractory epilepsy. In a retrospective study of 10 implanted epilepsy patients suffering also from migraine, eight had at least a $50 \%$ reduction in headache frequency six months after starting VNS (40). Other observational VNS studies included small numbers of patients, but reported overall an improvement in $50 \%$ of patients (32).

\section{Non-invasive PNS}

Compared to the invasive methods described above, noninvasive PNS can in theory be applied to any patient, including the less-disabled ones.

Transcutaneous supraorbital nerve stimulation (tSNS). The effectiveness of the portable tSNS Cefaly ${ }^{\circledR}$ for migraine prophylaxis has been recently evaluated in a randomized, double-blind sham-controlled trial (41). Sixty-seven episodic migraineurs were treated with daily tSNS or sham sessions of 20 minutes for three months. Compared to baseline, the mean monthly number of migraine days had significantly decreased after three months in the tSNS $(6.94 \pm 3.04$ vs. $4.88 \pm 3.46 ; p<0.05)$, but not in the sham group $(6.54 \pm 2.61 \quad$ vs. $6.22 \pm 2.99 ; \quad p=n s)$. The $50 \%$ responder rate was significantly greater in the tSNS $(38.1 \%)$ than in the sham group $(12.1 \%$, $p<0.05)$. Acute antimigraine drug intake was also significantly reduced in the verum but not in the sham group.

In an Internet survey of participants in the general population renting the tSNS Cefaly ${ }^{\circledR}$ device before deciding to buy it or not (42), a majority $(54.4 \%)$ of 2313 individuals declared themselves satisfied and decided to keep the device (average testing period: 58.2 days). Among the unsatisfied patients (46.6\%), the built-in monitoring system of the time the device was used showed poor compliance (tSNS used 48.6\% of the recommended time, $4.46 \%$ of patients did not even switch the device on). Only $4.3 \%$ of individuals reported one or more adverse event(s) such as local pain/intolerance to paresthesia $(2.03 \%)$, arousal changes $(0.82 \%)$, and headache after the stimulation $(0.52 \%)$. A transient local skin allergy was seen in $0.09 \%$.

There is no RCT of this device for the abortive treatment of migraine attacks.

Transcutaneous VNS. New devices thought to stimulate the vagus nerve transcutaneously (tVNS) have recently been developed and their efficacy as acute and preventive treatments of primary headaches is being evaluated. Preliminary open data in 30 patients suggest that the Gammacore $^{\circledR}$ device targeting the tVNS in the neck was effective in aborting migraine attacks, $21 \%$ of patients being pain free at two hours (43).

In a randomized, sham-controlled pilot study of chronic migraine patients (44), two months of three daily 90 -second stimulations produced at least a $50 \%$ reduction in headache days in four out of 26 patients $(15 \%)$, compared to none out of 23 in the sham-stimulated group. This modest beneficial effect was confirmed in the subsequent open-label phase (45), but further studies are clearly needed to determine the role of tVNS in migraine management. Besides neck muscle contractions in some patients, there were no significant adverse effects.

\section{Conclusion}

Invasive ONS still awaits definitive proof of efficacy and could be envisaged only after failure or intolerance of several preventive antimigraine drugs in chronic migraine sufferers (46). In medication overuse headache patients, it is crucial to detoxify before considering any invasive neurostimulation, as drug overuse seems to be 
associated with a less favorable outcome with ONS (47). Patients must be aware that outcome is uncertain, and that improvement may be moderate.

Noninvasive PNS, especially tSNS, can be proposed to less-disabled migraineurs as preventive or add-on migraine therapy (42). Although evidence of benefit is lacking, tSNS and tVNS could also be proposed as addon to drug-resistant chronic migraine patients, before turning to more invasive and expensive devices (48).

Because of the paresthesias they produce, it is notoriously difficult to correctly blind PNS trials. Appropriate RCTs are mandatory, the more so that the placebo response is greater with devices than with oral drug therapies (49). More studies are needed to verify that subthreshold stimulations can be adequately used as controls and to establish dose-response curves $(32,35)$.

The studies performed up to now with the noninvasive devices indicate that compliance may be the real challenge to solve in RCTs and in clinical practice (45).

\section{Infiltrations/blocks of pericranial nerves}

Many studies have been performed in the past decades to evaluate the effects of infiltrations or "blocks" in the region of the greater occipital nerve (GON) in migraine, both as preventive or acute treatment. The rationale for such treatments is the anatomo-physiological convergence of $\mathrm{C} 2$ dermatoma and trigeminovascular afferents in the spinal trigeminal nucleus underlying referred pain from the neck and orbitofrontal regions innervated by the ophthalmic nerve (50).

The major studies are summarized in Table 1 (51-62). Unfortunately, there are very few controlled trials and no standardized methods were used for the selection of migraine patients (some had fixed unilateral headache, others not), the timing of infiltrations (ictal or interictal), the technique of infiltrations or blocks (unilateral or bilateral, association with blocks of other pericranial nerves or trigger point injections, one or more interventions), the compounds used for the blocks (local anesthetics alone or combined with different types and dosages of steroids) and particularly the evaluation of outcomes (number of headache-free days, variable percentage reduction of headache days or attacks, non-uniformly standardized pain indices). The great heterogeneity of published studies therefore renders their evaluation difficult.

Overall, a complete or partial beneficial preventive effect was reported in $48-100 \%$ of adult migraineurs, lasting from a few days to several months. A retrospective study also found partial benefit $(<35 \%)$ from GON injections in pediatric chronic migraineurs (60). In one controlled, single-blinded study the addition of steroids for GON blocks was not superior to the anesthetic drug alone (56). Similarly, in one recent placebo-controlled, randomized, double-blinded trial (61) comparing GON injections with $2.5 \mathrm{ml} 0.5 \%$ bupivacaine plus $0.5 \mathrm{ml}$ (20 mg) methylprednisolone to blocks with $2.75 \mathrm{ml}$ normal saline plus $0.25 \mathrm{ml} 1 \%$ lidocaine in episodic $(n=54)$ and chronic $(n=9)$ migraineurs (verum group $n=33$; placebo group $n=30$ ), the blocks with steroids showed no superiority over placebo.

By contrast, in another recent double-blind, placebocontrolled study of suboccipital blocks in 72 chronic migraine patients - available at present only in abstract form - comparing four weekly injections of saline to $0.5 \%$ bupivacaine (62), the number of headache days decreased from $16.9 \pm 5.7$ days to $13.2 \pm 6.7$ days in the former $(p=0.035)$ but from $18.1 \pm 5.3$ days to $8.8 \pm 4.8$ days $(p<0.001)$ in the latter, and the superior effect of bupivacaine was confirmed in an open two-month extension study. Medication overuse tripled the risk of failure of the treatment in one study (58) but had no influence on outcome in two other ones $(55,59)$. In many studies palpation tenderness in the GON region was taken intuitively as a criterion for selecting patients for GON blockade/infiltration. The positive predictive value of GON tenderness was assessed in two independent studies with divergent results: It was associated with better outcome in the first (55), but not in the second (59).

GON blocks were also tested as a symptomatic treatment for acute migraine headache. Combined lidocaine blockade of GON and supraorbital (SO) nerves in 14 patients produced only negligible pain reduction $(6 \%)$ after 30 minutes; $50 \%$ of patients did not respond at all (63). By contrast, in an open study of 25 patients with unilateral migraine (11 episodic and 14 chronic) and brush allodynia, a GON block with $1 \mathrm{cc}$ of a $50 / 50$ mixture of $2 \%$ lidocaine and $0.5 \%$ bupivacaine reduced headache intensity by a mean of $46.8 \%$ in $89.5 \%$ of patients after 20 minutes and ipsilateral allodynia by a mean of $65.7 \%$ in all patients (64). The same procedure reduced the pain and allodynia scores respectively by $64 \%$ and $75 \%$ after five minutes (65). The mean duration of benefit in these studies was four days, both in episodic and chronic migraineurs. In a case report, a woman affected by basilar migraine underwent GON infiltration with $3 \mathrm{ml}$ of $0.25 \%$ bupivacaine and $1 \mathrm{ml}$ of $40 \mathrm{mg} / \mathrm{ml}$ triamcinolone and reported after a few minutes partial resolution of the aura symptoms and complete disappearance of the headache (66).

It is difficult to assess whether SO nerve blocks are effective, as they have rarely been studied in isolation. In the study by Bovim and Sand (1992) (63), the combination of GON and SO nerve blocks with $0.5-1.5 \mathrm{ml}$ of lidocaine $(20 \mathrm{mg} / \mathrm{ml}$ and $12.5 \mu \mathrm{g} / \mathrm{ml}$ adrenaline) was not effective. By contrast, in another study comparing $\mathrm{SO}$ and GON blocks alone and the combination of 


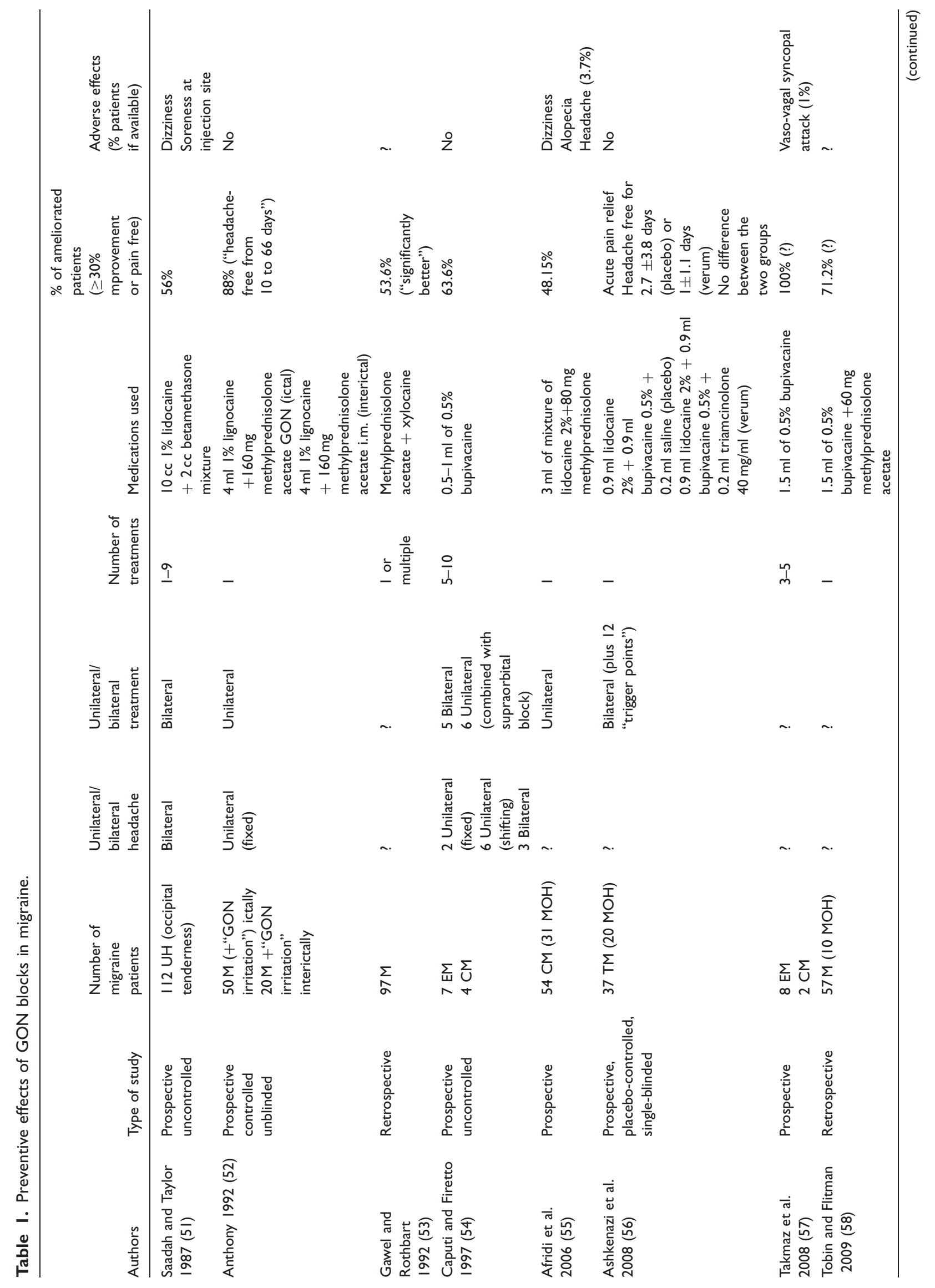




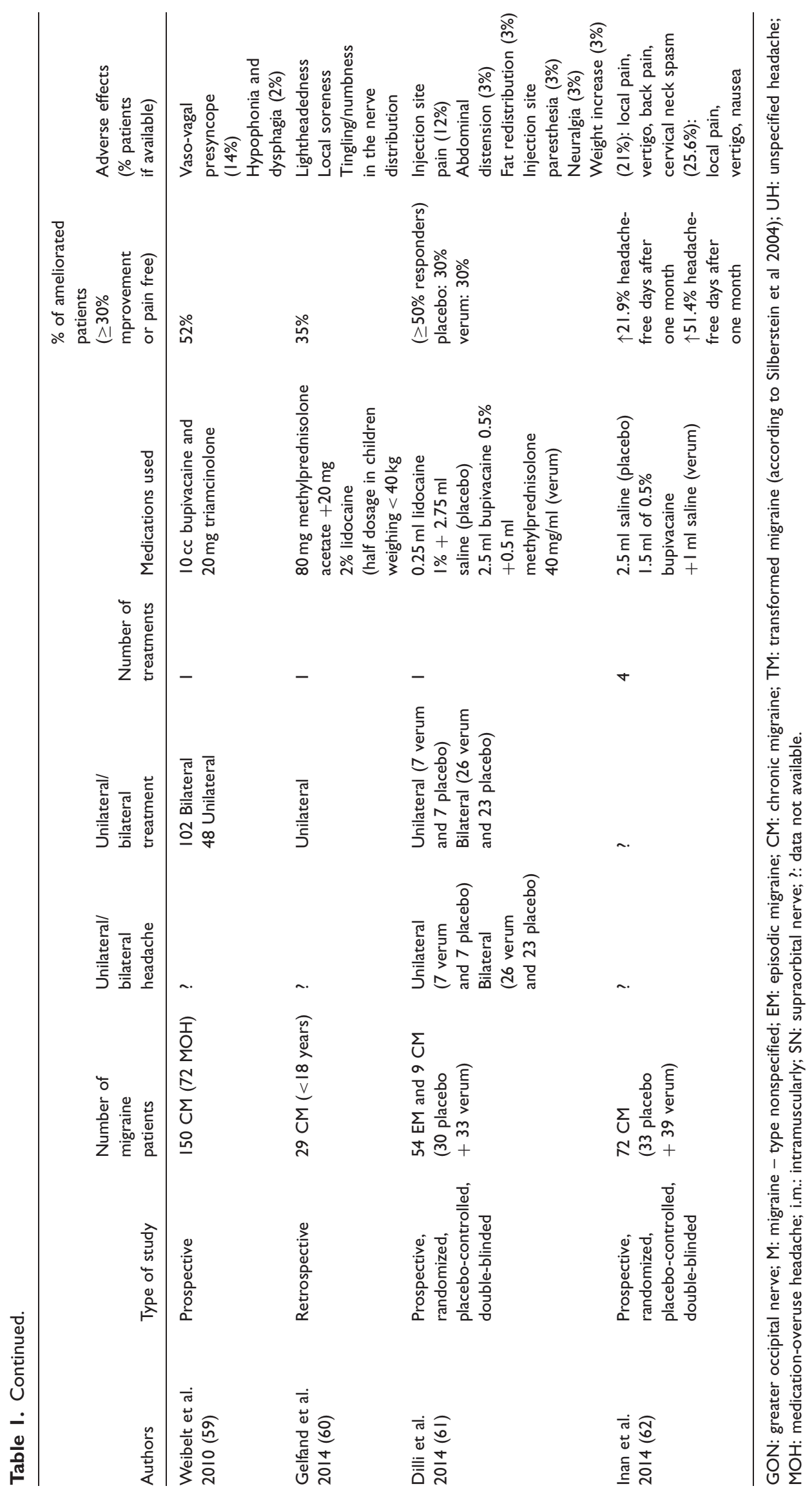


both, after SO blocks solely (54) a 50\% improvement of headache frequency was found in $68.75 \%$ of patients after one month and in $75 \%$ of patients after six months. Recently, three consecutive bilateral SO and infraorbital nerve blocks with $1.5 \mathrm{ml}$ of $1 \%$ lidocaine in episodic migraine patients were reported to significantly reduce mean headache frequency and MIDAS score (67).

To conclude, although infiltrations with steroids and anesthetic blocks of cranial nerves have been extensively used in migraine patients for a long time and effectiveness was reported in many observational studies, there are only two RCTs available: in one of them, comparing saline to bupivacaine in chronic migraine (62), the effect of bupivacaine exceeds that of placebo, but in the other, where bupivacaine plus methylprednisolone was compared to saline plus lidocaine, both groups had similar outcomes. Adverse effects reported after peripheral nerve blocks are rare and minor (see Table 1). Further studies are needed to determine in which subgroups of migraine patients suboccipital or SO nerve blocks are superior to placebo.

\section{Surgical decompressions}

As mentioned above, there is scarce evidence that nervous structures in the pericranium or face play a primary causative role in migraine pathogenesis. Many patients nevertheless refer their pain to the surface of the head or neck in close anatomical relationship with branches of pericranial nerves. Although these superficial pain locations are nowadays merely attributed to referred pain from the visceral part of the ophthalmic nerve, surgical attempts to treat migraine by acting directly on cranial nerves have been published as case reports as early as the first half of the 20th century (68).

More recently, decompression of pericranial nerves by sectioning neighboring muscles and sections of superficial nerve branches were proposed as therapeutic options for migraine patients. The bulk of data concerning these procedures originates from Guyuron's group in Cleveland (see Table 2) (69-81). These authors first studied retrospectively patients who underwent resection of corrugator supercilii muscle for rejuvenation and reported improving or disappearance of their headaches (69). This was followed by a prospective study on 29 patients with hypertrophy of the corrugator supercilii muscle and at least $50 \%$ amelioration by one injection of $25 \mathrm{U}$ of botulinum toxin type A into this muscle (70). Twenty-four patients out of 29 responded to botulinum toxin, 16 having complete disappearance of headaches, and eight partial improvements. The complete responders underwent a corrugator supercilii muscle resection while the others had in addition a transection of the zygomaticotemporal branch of the trigeminal nerve and repositioning of the temple soft tissues. After follow-up (222-494 days), all patients except one improved; 10 out of 22 operated patients were pain free. Assuming that various pericranial trigger sites might play a role in migraine and that they can be identified by local botulinum toxin injections, the same group in a subsequent unblinded prospective study (71) allocated patients to one of a combination of four surgical techniques and followed a nonoperated on control group of 25 patients for one year. Depending on the origin of pain and botulinum toxin effect, the surgical procedures were 1) removal of corrugator supercilii, depressor supercilii and procerus muscles (90\% of patients); 2) endoscopic removal of $3 \mathrm{~cm}$ of the zygomaticotemporal branch of the trigeminal nerve $(80 \%) ; 3)$ resection of the semispinalis capitis muscle and shielding of the greater occipital nerve $(38 \%) ; 4)$ septoplasty and inferior and/or middle turbinectomies $(70 \%)$. Ninety-two percent of the operated patients were reported to have at least $50 \%$ reduction in migraine headache frequency and duration, while only $15.8 \%$ of controls improved. There was also a significant effect on quality of life, work loss and cost for migraine care in the operated group.

In the five-year follow-up of the patients included in this study (75), out of 69 patients who completed it without re-interventions, six were operated at one site, 15 at two, 30 at three and 18 at all four trigger sites. Eighty-eight percent of patients reported beneficial effects from the surgical treatment at the end of the five-year follow-up: disappearance of headache in $29 \%, a \geq 50 \%$ reduction in $59 \%$.

In the only sham-controlled study (74), 130 patients with "frequent moderate to severe migraine" with or without aura were included on the basis that they reported a so-called "trigger site," i.e. a predominant site "where the migraine headache begins and settles and corresponds to the anatomical zone of potential irritation of the trigeminal nerve." Among them, 75 were included in the study because they had at least $50 \%$ amelioration after injection of $25 \mathrm{U}$ botulinum toxin into the "trigger" area and completed a one-year follow-up. They were divided into three groups according to the localization of the "trigger" site: frontal, temporal or occipital. The "frontal" group underwent the above-mentioned procedure 1, the "temporal" group procedure 2, and the "occipital" group procedure 3. In each group a third of patients underwent a sham operation. In the verum arm $(n=49) 83.7 \%$ of participants from the three groups reported a significant amelioration or elimination of headaches, whereas in the sham arm $(n=26) 57.7 \%$ had a similar positive outcome.

More recently, the same group published several retrospective studies aiming at identifying predictors 


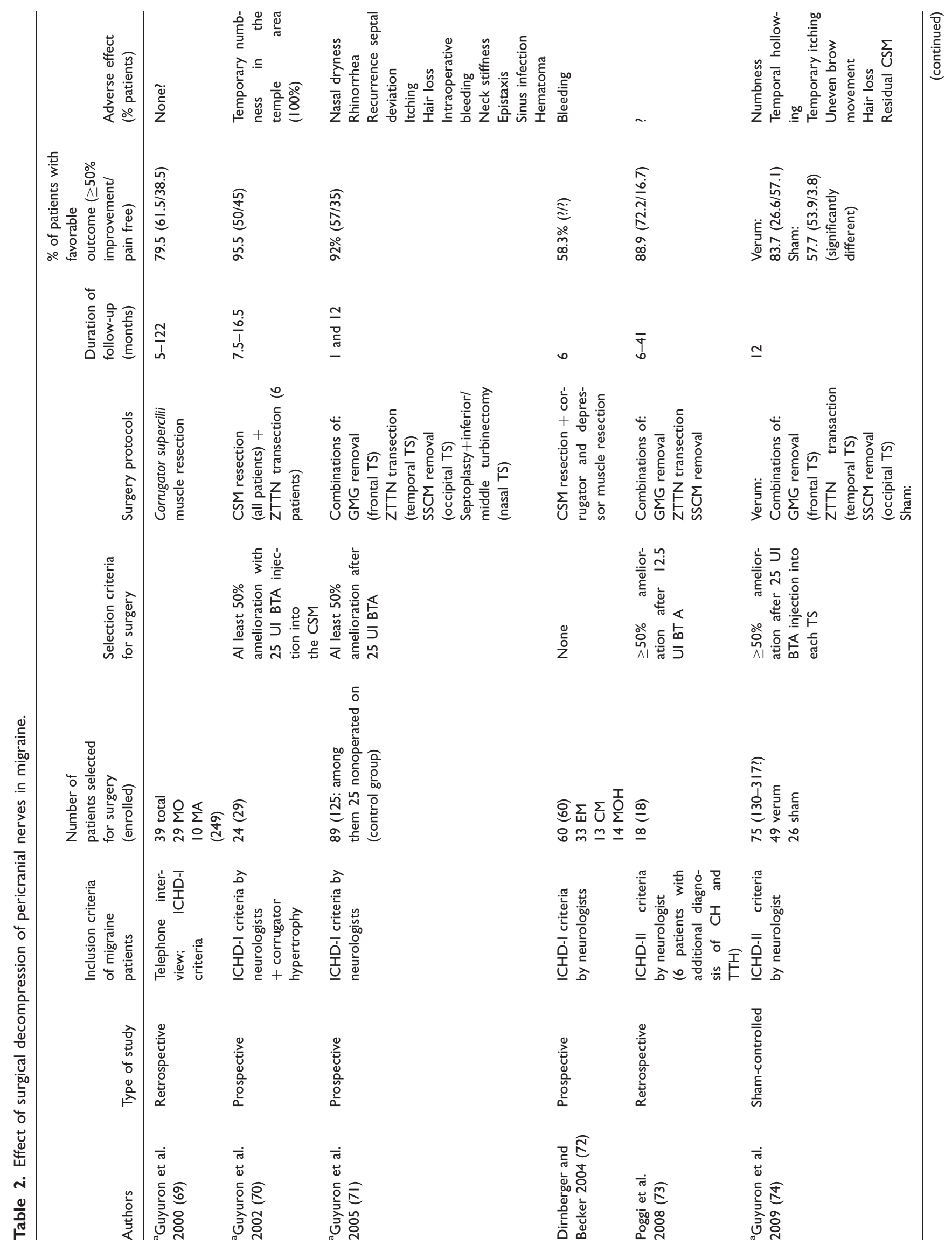




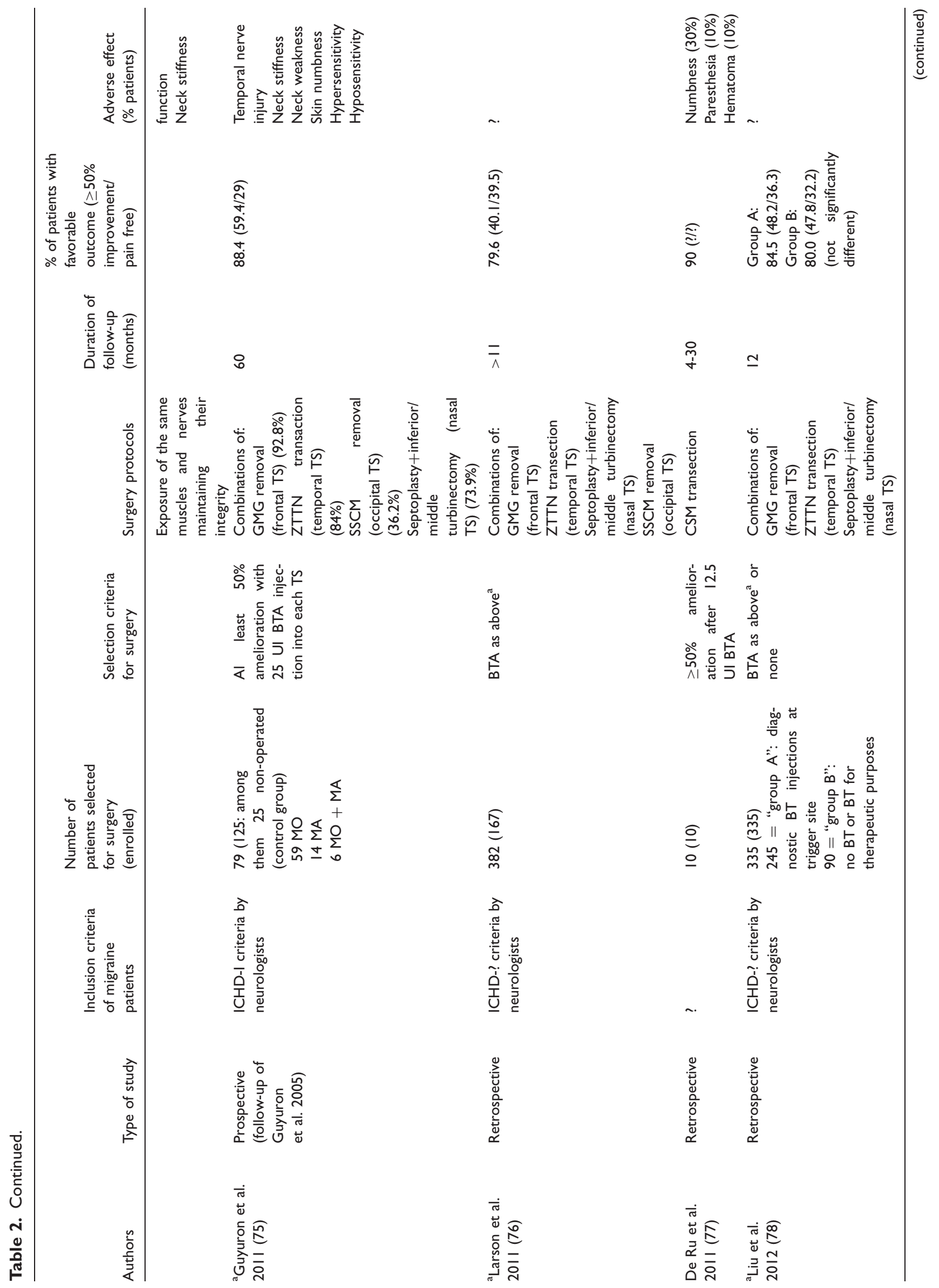




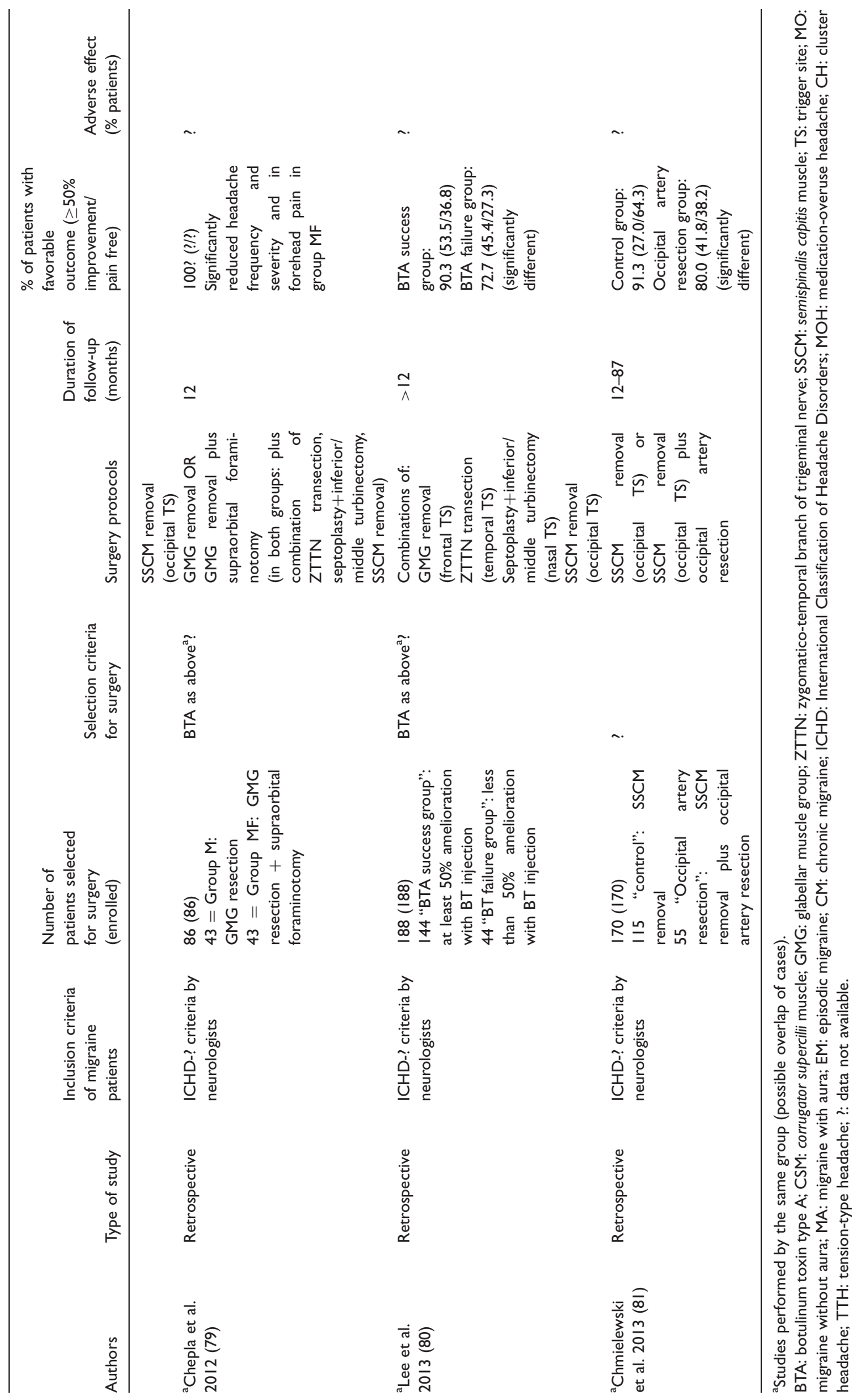


of favorable outcome $(76,78,80)$ or added value of additional surgical procedures like supraorbital foraminotomy (79) or ligation of the occipital artery (81).

Only three other groups have published retrospective studies on the effects of surgery in the frontal area in migraine. In the largest study (72) resection of the corrugator and depressor muscles in 60 episodic or chronic migraineurs, a $\geq 50 \%$ reduction in headache days at a six-month-follow-up was found in $58.3 \%$ of patients, most of them having a mild form of migraine. In another study (73) 16 out of 18 migraine patients responding to botulinum toxin and operated on at multiple sites in various combinations had a $\geq 50 \%$ amelioration of migraine headaches at a mean follow-up of 16 months. In 10 patients suffering from frontally located "chronic daily headache" (77) and ameliorated by $\geq 50 \%$ after at least two frontal injections of botulinum toxin, nine had $\geq 50 \%$ improvement after corrugator muscle resection.

The rationale for surgical decompression of pericranial nerves in migraine is that compression of peripheral nerves induces inflammation and peptide release that may reach the meninges and hence trigger migraine headaches. Though still speculative, this rationale may gain support from recent studies demonstrating extracranial projections of meningeal afferents (22).

One weakness of peripheral nerve decompressions is that the evidence comes from retrospective or prospective uncontrolled studies with only one exception. The migraine patients included in these studies all had some uncommon clinical characteristics (82), such as strictly localized and side-locked headache and the presence of "trigger sites" that are more typical of cranial neuralgias and tension-type headaches (4). Also, the "constellation of symptoms" by which the authors classify patients in order to operate on specific sites are common to other primary or secondary headaches like neuralgias, headaches attributed to temporomandibular dysfunction, chronic tension-type headache, chronic/recurring rhinosinusitis, mucosal contact points or concha bullosa, and whiplash. Many of these head pains may coexist with and/or aggravate migraine and their alleviation could indirectly cause clinical improvement in migraineurs.

Another puzzling observation in published studies concerns the selection of patients. Most of them were diagnosed as episodic migraineurs. Botulinum toxin type A injections at "trigger sites" reduced headache frequency by at least $50 \%$ in $58 \%$ and $90 \%$ of patients $(70,71,74,75)$, which clearly contrasts with the much lower responder rate (maximum 30\%, not significantly different from placebo) in RCTs of botulinum toxin in episodic migraine (83). As the placebo response has been very high in all botulinum toxin trials $(84,85)$, this may have favored the selection of placebo responders.
In the sole sham-controlled study of peripheral nerve decompression, the placebo response was particularly high $(58 \%)$, possibly because of the possibility that the incision and the undermining of pericranial tissues may have altered neurosensory functions and that some patients may have exaggerated their preoperative symptoms to increase their chance of selection for surgery $(70,71,74,77)$. In fact, the placebo response is in general higher with invasive than with drug treatments (49). The resulting therapeutic gain of $\pm 25 \%$ may appear clinically useful in very disabled patients, but in most published decompression studies there is no information about concomitant or previous preventive drug treatments or refractoriness to them.

Because of these confounding and atypical features surgical decompression of PNS cannot be considered as an established treatment option for migraineurs in general, although it may be useful in a subgroup of patients after careful weighing of possible side effects (see Table 2) and cost. Independent sham-controlled studies coming from different research groups and using standardized procedures for patient selection are warranted, including comparisons with optimized medical therapy.

\section{Conclusions}

Acting on pericranial nerve branches to treat migraine is not novel. Although current knowledge of migraine pathophysiology favors a central nervous system dysfunction (12), there is some evidence that interventions targeting PNS are able to modulate neuronal circuits involved in central sensitization and pain control. Invasive or noninvasive neurostimulation, anesthetic/ steroid blocks, and surgical decompression of pericranial nerves may act in this way, which suggests that their effect is merely symptomatic.

Advances in this therapeutic area will come from a better knowledge of migraine pathophysiology, more precise phenotyping of patients and advances in technology and treatment protocols. Larger, betterdesigned and comparative trials are needed to appraise the respective advantages, disadvantages and indications of most interventions discussed here. Such trials may be difficult to set up for treatments like nerve blocks for which there is no commercial interest.

The challenge will be to use comparable standards for the evaluation of their effects in drug trials and to manage adequately the blinding caveat.

\section{Literature search methods}

English-language publications were searched for in PubMed up to July 2014, and updated in December 2014. 
The following search terms were used: "migraine neurostimulation," "migraine neuromodulation," "migraine injection," "migraine nerve infiltrations," "migraine nerve blocks," "migraine surgery" and "migraine decompression." All the identified publications were individually assessed according to their relevance to the topic. Specific exclusion criteria included: publications on single case reports, editorials and other review articles unless of exceptional importance. The reference lists of identified publications were also scrutinized for further relevant publications.

\section{Clinical implications}

- The definitive evidence that percutaneous occipital nerve stimulation (ONS) is effective in chronic migraine has not been obtained yet, but some randomized controlled trials (RCTs) indicate nonetheless that ONS might ameliorate a subgroup of patients.

- Noninvasive transcutaneous supraorbital neurostimulation is superior to sham stimulation for the prevention of episodic migraine, and noninvasive vagus nerve stimulation in the neck is promising for chronic migraine. Technological advances and improved stimulation protocols may improve performance of these methods in the near future. Further study results are eagerly awaited.

- Since it is inexpensive and safe and there are some indications from observational studies for its usefulness, suboccipital infiltration may be an add-on option in selected patients, in particular those with fixed unilateral headaches and ipsilateral autonomic symptoms. Convincing evidence for efficacy from RCTs is, however, missing.

- Surgical decompression of pericranial nerves was found superior to sham surgery in one study and most published case reports are from the same group. Because of the heterogeneity of patients included, selection bias and the questionable inclusion criteria, the efficacy and usefulness of surgical decompressions in migraine patients cannot be considered as definitely established until other RCTs from other independent groups confirm it.

\section{Funding}

This research received no specific grant from any funding agency in the public, commercial, or not-for-profit sectors.

\section{Conflict of interest}

A.A., C.D.A. and D.M. have nothing to declare.

J.S. is an advisor for Cefaly-Technology, St Jude Medical, ATI, Medtronic, AMGEN and Gedeon Richter.

\section{References}

1. Merikangas KR. Contributions of epidemiology to our understanding of migraine. Headache 2013; 53: 230-246.

2. Buse D, Manack A, Serrano D, et al. Headache impact of chronic and episodic migraine: Results from the American Migraine Prevalence and Prevention study. Headache 2012; 52: 3-17.

3. Lipton RB, Stewart WF, Diamond S, et al. Prevalence and burden of migraine in the United States: Data from the American Migraine Study II. Headache 2001; 41: 646-657.

4. Headache Classification Committee of the International Headache Society (IHS). The International Classification of Headache Disorders, 3rd edition (beta version). Cephalalgia 2013; 33: 629-808.

5. Edvinsson L, Villalón CM and MaassenVanDenBrink A. Basic mechanisms of migraine and its acute treatment. Pharmacol Ther 2012; 136: 319-333.
6. Leao AAP. Spreading depression of activity in cerebral cortex. J Neurophysiol 1944; 7: 359-390.

7. Lauritzen M. Cortical spreading depression in migraine. Cephalalgia 2001; 21: 757-760.

8. Akerman S, Holland PR and Goadsby PJ. Diencephalic and brainstem mechanisms in migraine. Nat Rev Neurosci 2011; 12: 570-584.

9. Giffin NJ, Ruggiero L, Lipton RB, et al. Premonitory symptoms in migraine: An electronic diary study. Neurology 2003; 60: 935-940.

10. Kelman L. The premonitory symptoms (prodrome): A tertiary care study of 893 migraineurs. Headache 2004; 44: 865-872.

11. Maniyar FH, Sprenger T, Monteith $T$, et al. Brain activations in the premonitory phase of nitroglycerin-triggered migraine attacks. Brain 2014; 137: 232-241.

12. De Tommaso M, Ambrosini A, Brighina F, et al. Altered sensory processing in migraine. Nat Rev Neurol 2014; 10: 144-155.

13. Montagna P, Cortelli P, Monari L, et al. 31P-magnetic resonance spectroscopy in migraine without aura. Neurology 1994; 44: 666-669.

14. Welch KM, Levine SR, D'Andrea G, et al. Preliminary observations on brain energy metabolism in migraine studied by in vivo phosphorus 31 NMR spectroscopy. Neurology 1989; 39: 538-541.

15. Reyngoudt H, Paemeleire K, Descamps B, et al. 31PMRS demonstrates a reduction in high-energy 
phosphates in the occipital lobe of migraine without aura patients. Cephalalgia 2011; 31: 1243-1253.

16. Sándor P, Ambrosini A, Agosti RM, et al. Genetics of migraine: Possible links to neurophysiological abnormalities. Headache 2002; 42: 365-377.

17. Sprenger T and Borsook D. Migraine changes the brain: Neuroimaging makes its mark. Curr Opin Neurol 2012; 25: 252-262.

18. Coppola $\mathrm{G}$ and Schoenen J. Cortical excitability in chronic migraine. Curr Pain Headache Rep 2012; 16: 93-100.

19. Lous I and Olesen J. Evaluation of pericranial tenderness and oral function in patients with common migraine, muscle contraction headache and 'combination headache'. Pain 1982; 12: 385-393.

20. Calandre EP, Hidalgo J, García-Leiva JM, et al. Trigger point evaluation in migraine patients: An indication of peripheral sensitization linked to migraine predisposition? Eur J Neurol 2006; 13: 244-249.

21. Seo BF, Jung SN, Sohn WI, et al. Lymph node compression of the lesser occipital nerve: A cause of migraine. J Plast Reconstr Aesthet Surg 2011; 64: 1657-1660.

22. Schueler M, Neuhuber WL, De Col R, et al. Innervation of rat and human dura mater and pericranial tissues in the parieto-temporal region by meningeal afferents. Headache 2014; 54: 996-1009.

23. Schueler M, Messlinger K, Dux M, et al. Extracranial projections of meningeal afferents and their impact on meningeal nociception and headache. Pain 2013; 154: 1622-1631.

24. Bigal ME and Lipton RB. Overuse of acute migraine medications and migraine chronification. Curr Pain Headache Rep 2009; 13: 301-307.

25. Whyte CA and Tepper SJ. Adverse effects of medications commonly used in the treatment of migraine. Expert Rev Neurother 2009; 9: 1379-1391.

26. Ferrari A, Leone S, Vergoni AV, et al. Similarities and differences between chronic migraine and episodic migraine. Headache 2007; 47: 65-72.

27. Zeeberg P, Olesen J and Jensen R. Discontinuation of medication overuse in headache patients: Recovery of therapeutic responsiveness. Cephalalgia 2006; 26: 1192-1198.

28. Melzack R and Wall PD. Pain mechanisms: A new theory. Science 1965; 150: 971-979.

29. DeSantana JM, Da Silva LF, De Resende MA, et al. Transcutaneous electrical nerve stimulation at both high and low frequencies activates ventrolateral periaqueductal grey to decrease mechanical hyperalgesia in arthritic rats. Neuroscience 2009; 163: 1233-1241.

30. Cruccu G, Aziz TZ, Garcia-Larrea L, et al. EFNS guidelines on neurostimulation therapy for neuropathic pain. Eur J Neurol 2007; 14: 952-970.

31. Weiner RL and Reed KL. Peripheral neurostimulation for control of intractable occipital neuralgia. Neuromodulation 1999; 2: 217-222.

32. Magis D and Schoenen J. Advances and challenges in neurostimulation for headaches. Lancet Neurol 2012; 11: 708-719.

33. Lipton R, Goadsby P, Cady R, et al. PRISM study: Occipital nerve stimulation for treatment-refractory migraine. Cephalalgia 2009; 29(Suppl 1): 30.
34. Saper JR, Dodick DW, Silberstein SD, et al. Occipital nerve stimulation for the treatment of intractable chronic migraine headache: ONSTIM feasibility study. Cephalalgia 2011; 31: 271-285.

35. Silberstein S, Dodick D, Saper J, et al. Safety and efficacy of peripheral nerve stimulation of the occipital nerves for the management of chronic migraine: Results from a randomized, multicenter, double-blinded, controlled study. Cephalalgia 2012; 32: 1165-1179.

36. Dodick D, Silberstein S, Huh B, et al. Evidence for longterm efficacy of peripheral nerve stimulation of occipital nerves in the management of chronic migraine. Cephalalgia 2013; 33(Suppl): 58.

37. Slotty P, Bara G, Kowatz L, et al. Occipital nerve stimulation for chronic migraine: A randomized trial on subthreshold stimulation. Cephalalgia 2015; 35: 73-78.

38. Reed KL, Black SB, Banta CJ 2nd, et al. Combined occipital and supraorbital neurostimulation for the treatment of chronic migraine headaches: Initial experience. Cephalalgia 2010; 30: 260-271.

39. Reed KL, Will KR, Chapman J, et al. Combined occipital and supraorbital neurostimulation for chronic migraine headaches: An extended case series. Cephalalgia 2011; 31(Suppl 1): 98.

40. Lenaerts ME, Oommen KJ, Couch JR, et al. Can vagus nerve stimulation help migraine? Cephalalgia 2008; 28: 392-395.

41. Schoenen J, Vandersmissen B, Jeangette $S$, et al. Migraine prevention with a supraorbital transcutaneous stimulator: A randomized controlled trial. Neurology 2013; 80: 697-704.

42. Magis D, Sava S, d'Elia TS, et al. Safety and patients' satisfaction of transcutaneous supraorbital neurostimulation (tSNS) with the Cefaly ${ }^{\circledR}$ device in headache treatment: A survey of 2,313 headache sufferers in the general population. J Headache Pain 2013; 14: 95.

43. Goadsby P, Grosberg B, Mauskop A, et al. Effect of noninvasive vagus nerve stimulation on acute migraine: An open-label pilot study. Cephalalgia 2014; 34: 986-993.

44. Silberstein SD, Neves da Silva A, Calhoun AH, et al. Non-invasive vagus nerve stimulation for chronic migraine prevention in a prospective, randomized, sham-controlled pilot study (the EVENT study): Report from the double-blind phase. Late Breaking Poster 19 at the 56th Annual Scientific Meeting of the American Headache Society, Los Angeles, CA, USA, 26-29 June 2014.

45. Silberstein SD, Neves da Silva A, Calhoun AH, et al. Chronic migraine prevention with non-invasive vagus nerve stimulation in a prospective pilot study (the EVENT study): Report from the open-label phase. Late Breaking Poster 21 at the 56th Annual Scientific Meeting of the American Headache Society, Los Angeles, CA, USA, 26-29 June 2014.

46. Goadsby PJ, Schoenen J, Ferrari MD, et al. Towards a definition of intractable headache for use in clinical practice and trials. Cephalalgia 2006; 26: 1168-1170.

47. Paemeleire K, Van Buyten JP, Van Buynder M, et al. Phenotype of patients responsive to occipital nerve 
stimulation for refractory head pain. Cephalalgia 2010; 30: $662-673$.

48. Martelletti P, Jensen RH, Antal A, et al. Neuromodulation of chronic headaches: Position statement from the European Headache Federation. J Headache Pain 2013; 14: 86 .

49. Meissner K, Fassler M, Rucker G, et al. Differential effectiveness of placebo treatments: A systematic review of migraine prophylaxis. JAMA Intern Med 2013; 173: 1941-1951.

50. Kerr FWL. A mechanism to account for frontal headache in cases of posterior fossa tumour. J Neurosurg 1961; 18 : 605-609.

51. Saadah HA and Taylor FB. Sustained headache syndrome associated with tender occipital nerve zones. Headache 1987; 27: 201-205.

52. Anthony M. Headache and the greater occipital nerve. Clin Neurol Neurosurg 1992; 94: 297-301.

53. Gawel MJ and Rothbart PJ. Occipital nerve block in the management of headache and cervical pain. Cephalalgia 1992; 12: 9-13.

54. Caputi CA and Firetto V. Therapeutic blockade of greater occipital and supraorbital nerves in migraine patients. Headache 1997; 37: 174-179.

55. Afridi SK, Shields KG, Bhola R, et al. Greater occipital nerve injection in primary headache syndromes-prolonged effects from a single injection. Pain 2006; 122: 126-129.

56. Ashkenazi A, Matro R, Shaw JW, et al. Greater occipital nerve block using local anaesthetics alone or with triamcinolone for transformed migraine: A randomised comparative study. J Neurol Neurosurg Psychiatry 2008; 79: 415-417.

57. Takmaz SA, Inan N, Uçler S, et al. Greater occipital nerve block in migraine headache: Preliminary results of 10 patients. Agri 2008; 20: 47-50.

58. Tobin JA and Flitman SS. Occipital nerve blocks: Effect of symptomatic medication: Overuse and headache type on failure rate. Headache 2009; 49: 1479-1485.

59. Weibelt S, Andress-Rothrock D, King W, et al. Suboccipital nerve blocks for suppression of chronic migraine: Safety, efficacy, and predictors of outcome. Headache 2010; 50: 1041-1044.

60. Gelfand AA, Reider AC and Goadsby PJ. Outcomes of greater occipital nerve injections in pediatric patients with chronic primary headache disorders. Pediatr Neurol 2014; 50: $135-139$.

61. Dilli E, Halker R, Vargas B, et al. Occipital nerve block for the short-term preventive treatment of migraine: A randomized, double-blinded, placebo-controlled study. Cephalalgia. Epub ahead of print 12 December 2014. DOI: $10.1177 / 0333102414561872$.

62. Inan LE, Inan N, Karadas O, et al. Greater occipital nerve blocks with bupivacaine in the treatment of chronic migraine. Randomized, multicenter, double-blind, parallel, placebo-controlled study. Eur J Neurol 2014; 21(S1): 52.

63. Bovim $G$ and Sand T. Cervicogenic headache, migraine without aura and tension-type headache. Diagnostic blockade of greater occipital and supra-orbital nerves. Pain 1992; 51: 43-48.

64. Ashkenazi A and Young WB. The effects of greater occipital nerve block and trigger point injection on brush allodynia and pain in migraine. Headache 2005; 45: $350-354$.

65. Young W, Cook B, Malik S, et al. The first 5 minutes after greater occipital nerve block. Headache 2008; 48: 1126-1128.

66. Baron EP, Tepper SJ, Mays M, et al. Acute treatment of basilar-type migraine with greater occipital nerve blockade. Headache 2010; 50: 1057-1059.

67. Ilhan Alp S and Alp R. Supraorbital and infraorbital nerve blockade in migraine patients: Results of 6-month clinical follow-up. Eur Rev Med Pharmacol Sci 2013; 17: 1778-1781.

68. Knight G. Surgical treatment of migraine. Proc $R$ Soc Med 1962; 55: 172-176.

69. Guyuron B, Varghai A, Michelow BJ, et al. Corrugator supercilii muscle resection and migraine headaches. Plast Reconstr Surg 2000; 106: 429-434.

70. Guyuron B, Tucker T and Davis J. Surgical treatment of migraine headaches. Plast Reconstr Surg 2002; 109: 2183-2189.

71. Guyuron B, Kriegler JS, Davis J, et al. Comprehensive surgical treatment of migraine headaches. Plast Reconstr Surg 2005; 115: 1-9.

72. Dirnberger F and Becker K. Surgical treatment of migraine headaches by corrugator muscle resection. Plast Reconstr Surg 2004; 114: 652-657.

73. Poggi JT, Grizzell BE and Helmer SD. Confirmation of surgical decompression to relieve migraine headaches. Plast Reconstr Surg 2008; 122: 115-122.

74. Guyuron B, Reed D, Kriegler JS, et al. A placebo-controlled surgical trial of the treatment of migraine headaches. Plast Reconstr Surg 2009; 124: 461-468.

75. Guyuron B, Kriegler JS, Davis J, et al. Five-year outcome of surgical treatment of migraine headaches. Plast Reconstr Surg 2011; 127: 603-608.

76. Larson K, Lee M, Davis J, et al. Factors contributing to migraine headache surgery failure and success. Plast Reconstr Surg 2011; 128: 1069-1075.

77. de Ru JA, Schellekens PP and Lohuis PJ. Corrugator supercilii transection for headache emanating from the frontal region: A clinical evaluation of ten patients. J Neural Transm 2011; 118: 1571-1574.

78. Liu MT, Armijo BS and Guyuron B. A comparison of outcome of surgical treatment of migraine headaches using a constellation of symptoms versus botulinum toxin type A to identify the trigger sites. Plast Reconstr Surg 2012; 129: 413-419.

79. Chepla KJ, Oh E and Guyuron B. Clinical outcomes following supraorbital foraminotomy for treatment of frontal migraine headache. Plast Reconstr Surg 2012; 129: $656 \mathrm{e}-662 \mathrm{e}$.

80. Lee M, Monson MA, Liu MT, et al. Positive botulinum toxin type a response is a prognosticator for migraine surgery success. Plast Reconstr Surg 2013; 131: 751-757.

81. Chmielewski L, Liu MT and Guyuron B. The role of occipital artery resection in the surgical treatment of 
occipital migraine headaches. Plast Reconstr Surg 2013; 131: 351e-356e.

82. D'Amico D, Leone M and Bussone G. Side-locked unilaterality and pain localization in long-lasting headaches: Migraine, tension-type headache, and cervicogenic headache. Headache 1994; 34: 526-530.

83. Jackson JL, Kuriyama A and Hayashino Y. Botulinum toxin A for prophylactic treatment of migraine and tension headaches in adults: A meta-analysis. JAMA 2012; 307: 1736-1745.
84. Delstanche $\mathrm{S}$ and Schoenen J. Botulinum toxin for the treatment of headache: A promising path on a "dead end road"? Acta Neurol Belg 2010; 110: 221-229.

85. Dodick DW, Turkel CC, DeGryse RE, et al. OnabotulinumtoxinA for treatment of chronic migraine: Pooled results from the double-blind, randomized, placebo-controlled phases of the PREEMPT clinical program. Headache 2010; 50: 921-936. 